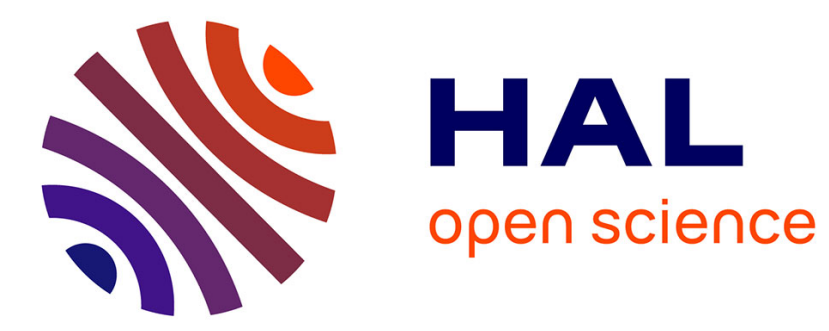

\title{
Performance of the liquid argon final calibration board
}

C. de La Taille, N. Seguin-Moreau, L. Serin, N. Dumont-Dayot, I.

Wingerter-Seez

\section{To cite this version:}

C. de La Taille, N. Seguin-Moreau, L. Serin, N. Dumont-Dayot, I. Wingerter-Seez. Performance of the liquid argon final calibration board. International Conference on Calorimetry in High Energy Physics

- CALOR2004 11, Mar 2004, Perugia, Italy. pp.143-150. in2p3-00021999

\section{HAL Id: in2p3-00021999 \\ https://hal.in2p3.fr/in2p3-00021999}

Submitted on 30 Jun 2004

HAL is a multi-disciplinary open access archive for the deposit and dissemination of scientific research documents, whether they are published or not. The documents may come from teaching and research institutions in France or abroad, or from public or private research centers.
L'archive ouverte pluridisciplinaire HAL, est destinée au dépôt et à la diffusion de documents scientifiques de niveau recherche, publiés ou non, émanant des établissements d'enseignement et de recherche français ou étrangers, des laboratoires publics ou privés. 


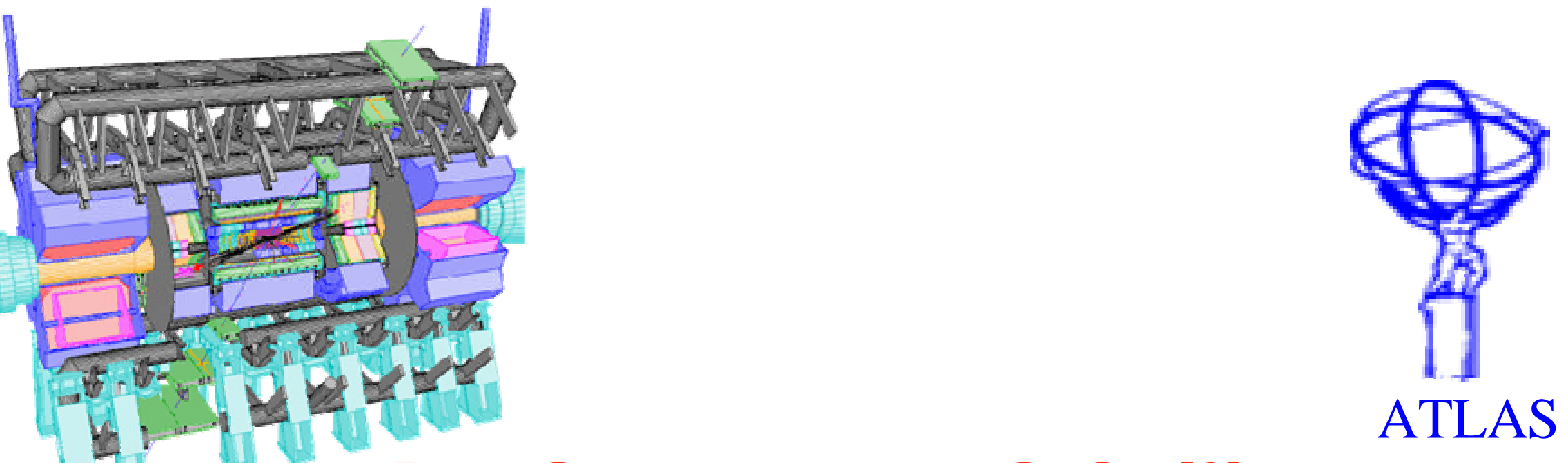

\section{Performance of Calib128 LArG final calibration board}

N. Dumont-Dayot, G. Ionescu, $\mathcal{N}$. Massol, I Wingerter-Seez LAPP Annecy

P. Imbert, C. de La Taille, $\mathcal{N}$. Seguin-Moreau, L. Serin $\mathcal{L A L}$ Orsay

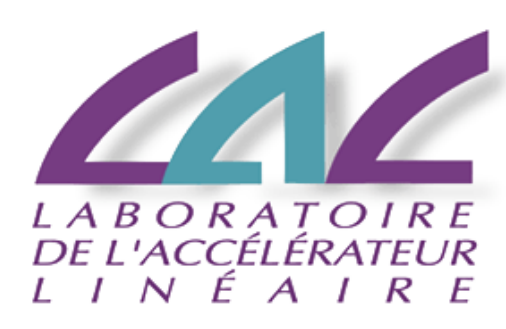


$\mathcal{A T L A S}$ Lar em calorimeter readout

ove?

\section{6 boards@128ch}
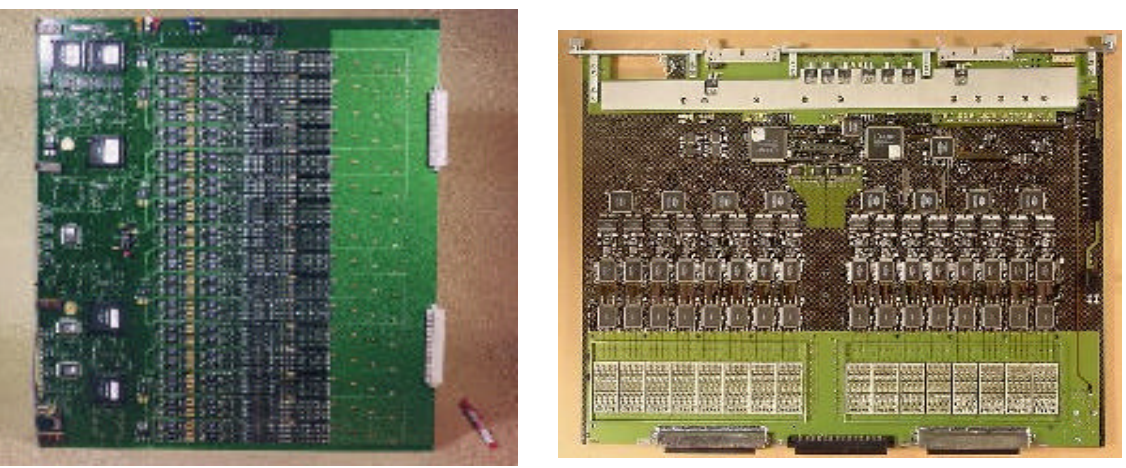

Front End Crate:

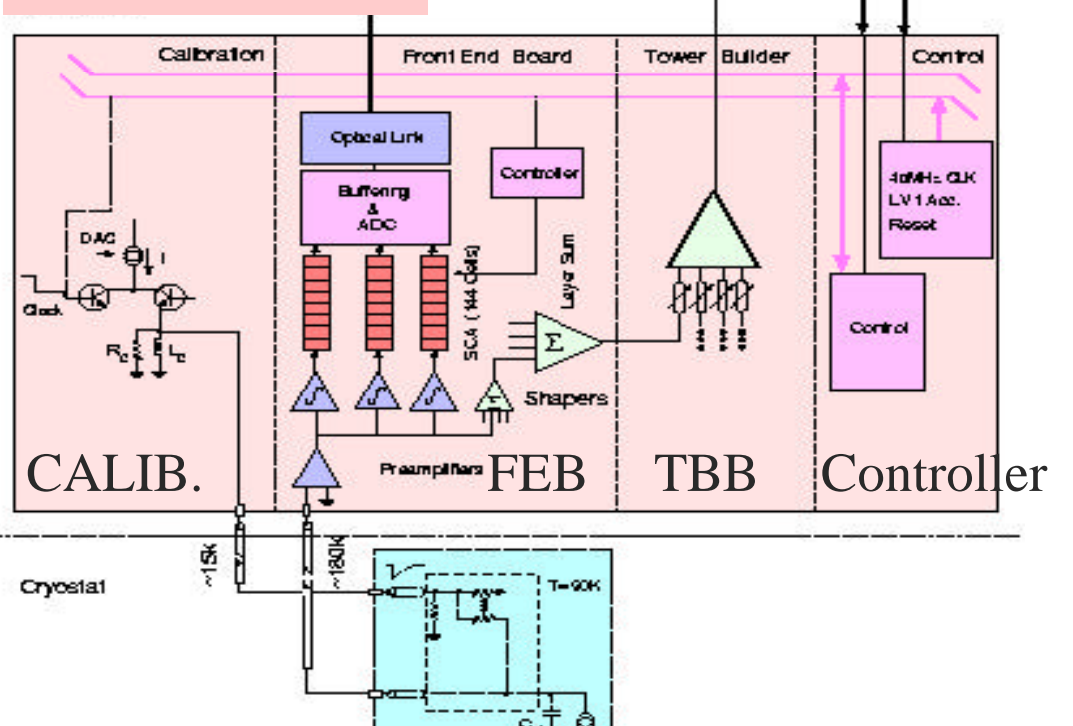

Front End Board (FEB) :

1524 boards@128ch

Readout and

Calib. signals

Cold to warm Feedthrough

Cryostat

1

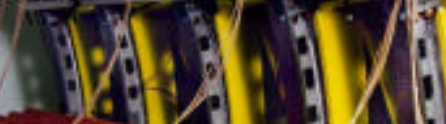
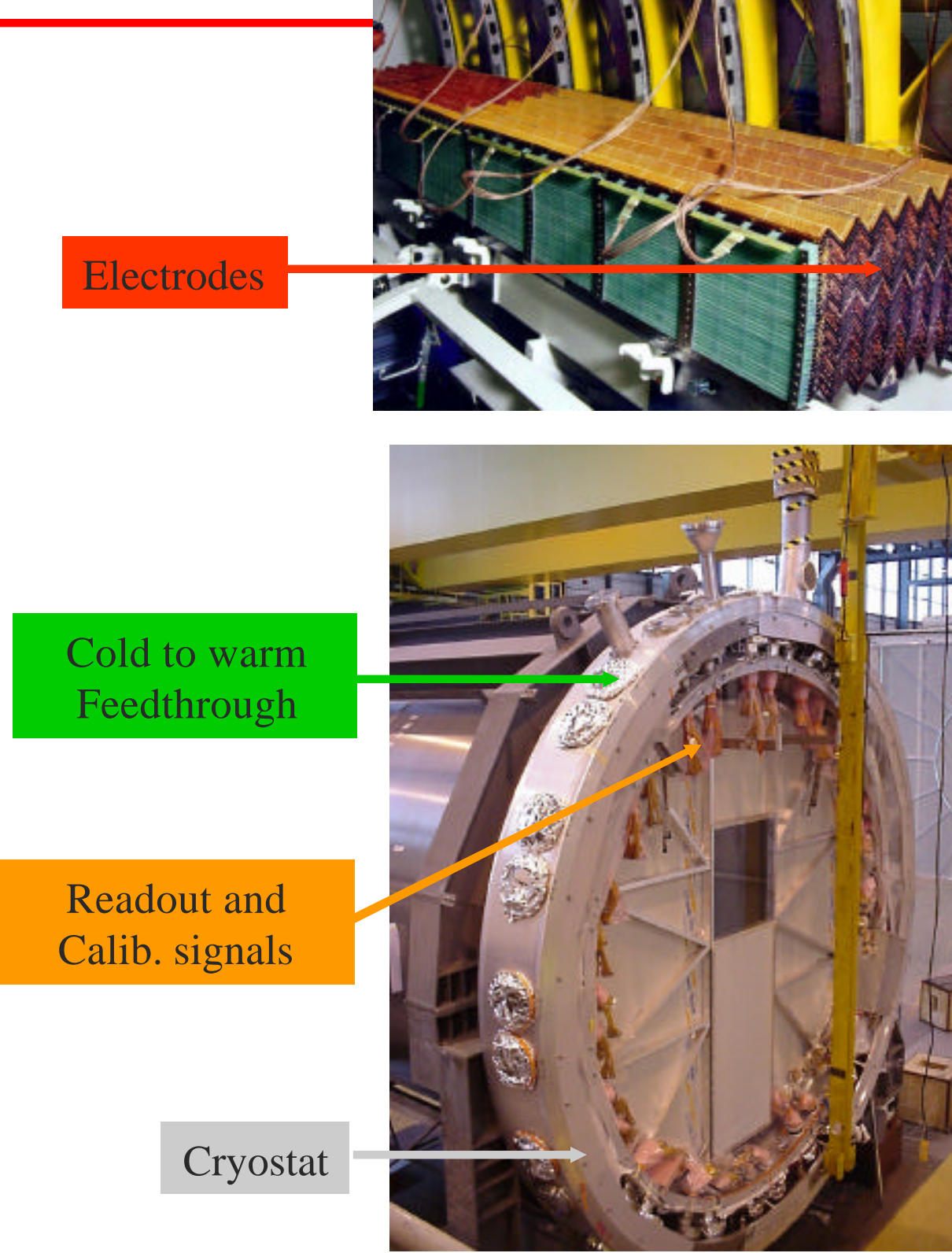
- Goal: Inject a precise current pulse as close as possible as the detector pulse

- Injection with precision resistors

- Rise time <1ns, Decay time $450 \mathrm{~ns}$. Dynamic range: 16 bits

- Output pulse: $100 \mu \mathcal{V}$ to $5 \mathcal{V}$ in 500

- Integral non line arity $<0.1 \%$.

- Uniformity between channels $<0.25 \%$

- To kee $p$ calorimeter constant term be low $0.7 \%$ )

- Timing between physics and calibration pulse \pm 1 ns

- Operation in around 100 Gauss field - Radiation frardness:

- $50 \mathrm{~Gy}, 1.610^{12}$ Neutrons $/ \mathrm{cm}^{2}$ in 10 years

- Qualification at $500 \mathrm{~Gy}, 1.610^{13}$ Neutrons $/ \mathrm{cm}^{2}$ to include safe ty factors Run at a few $\mathrm{KHz}$

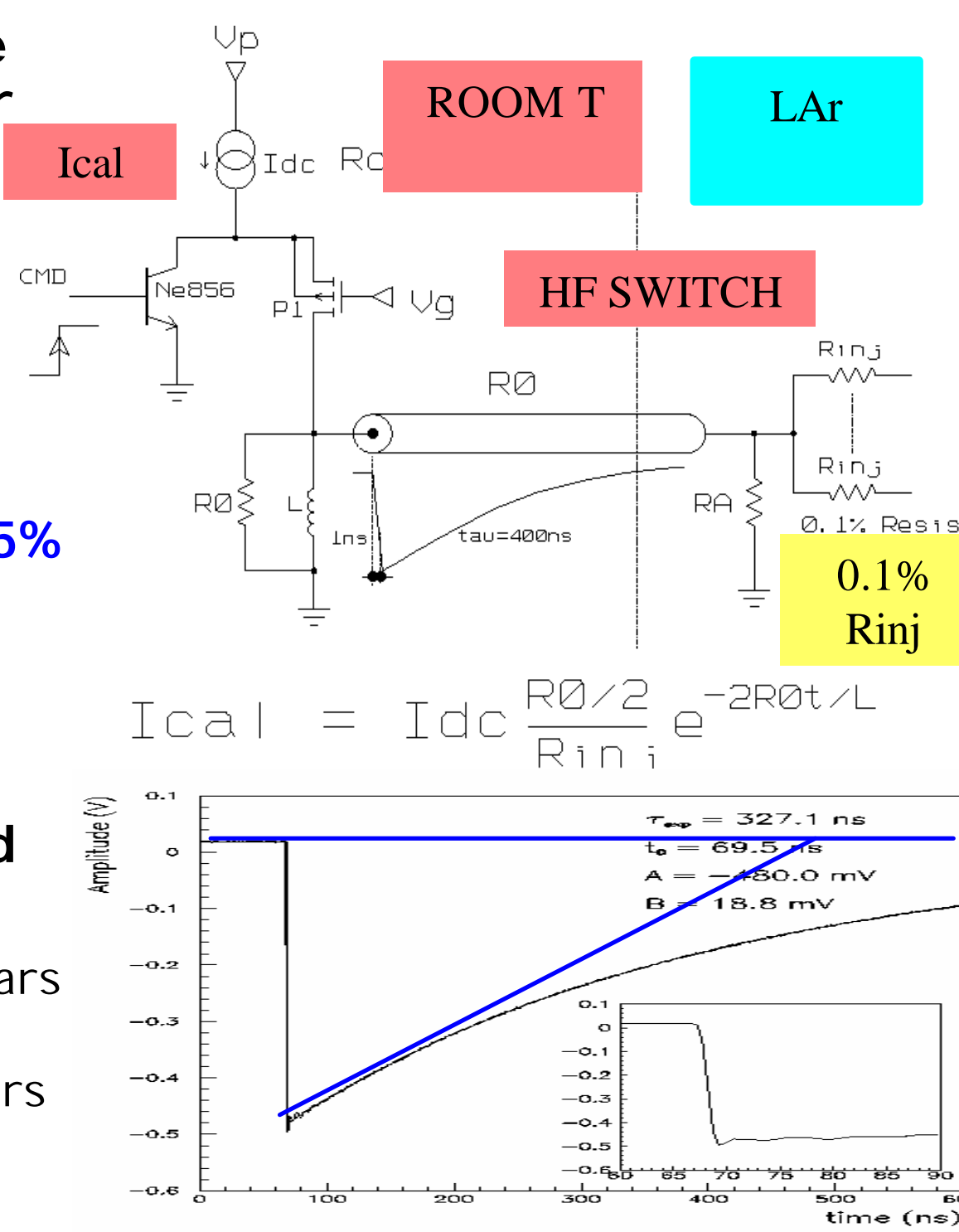


12 boards produced in 1998 with COTS for module 0

- 5 years successfuloperation in beam tests.

- Excellent uniformity :0.11\% rms on 1300 channels

- But radiation soft: COTS failed at $20 \mathrm{~Gy}$

Active elements designed in DMILL in 1999-2001

- $\mathcal{D A C}, \mathcal{P u l s e r}$, Controllogic, de lay chip

- Radiation qualified at $5 \mathrm{KGy}$

- Improved performance (DAC stability, parasitic signal at $\mathcal{D A C}=0, \mathcal{D A C}$ stability and offset)

- Simplified logic, 10 Alteras replaced by 6 identical AS ICS (DMM LL Calogic)

- All ASICs produced in 2003, currently under test

3 radiation frard boards produced in 2002-2003

- Final design review in 2002

- Production readiness revie w passed in march 2004

- Production of 140 boards in 2004-2005
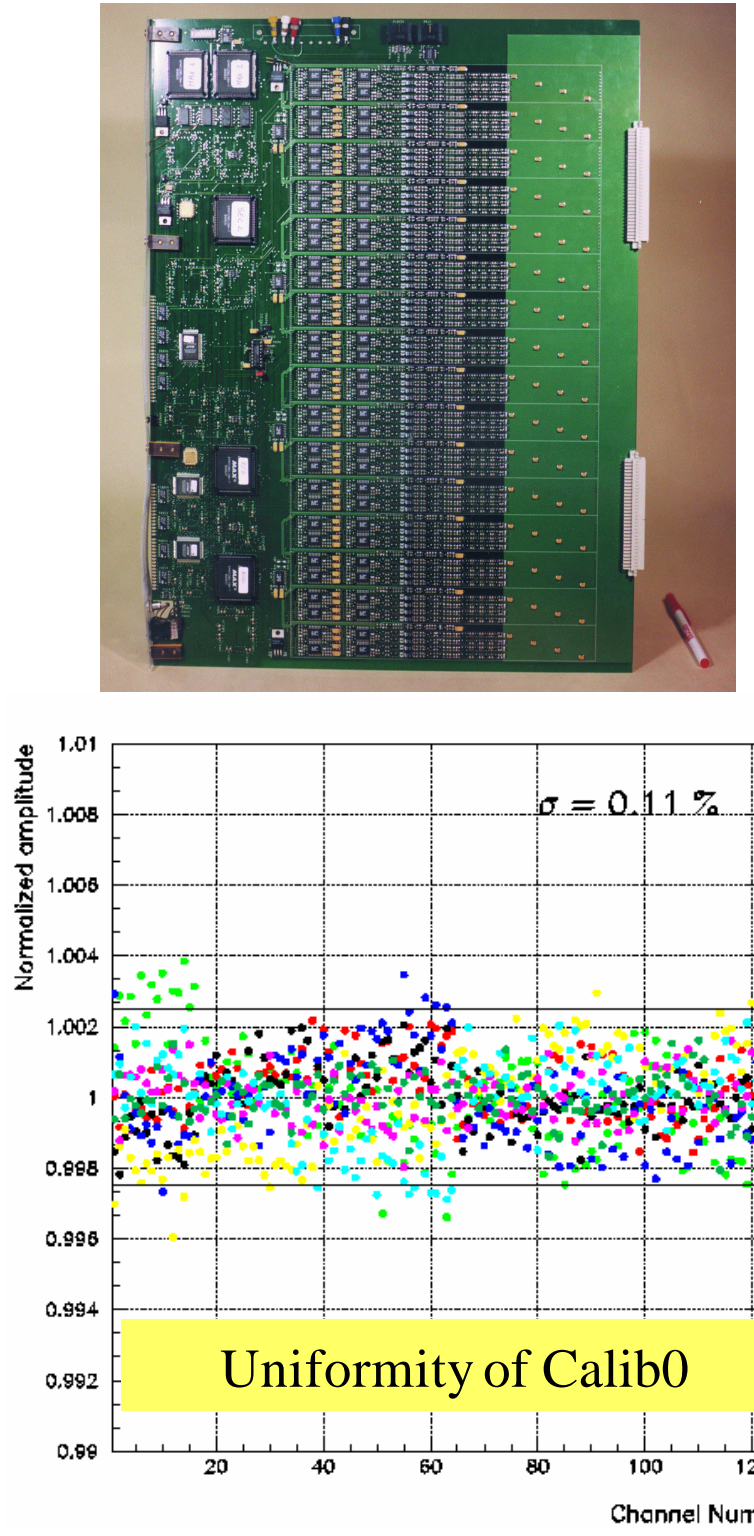
Final calibration board layout

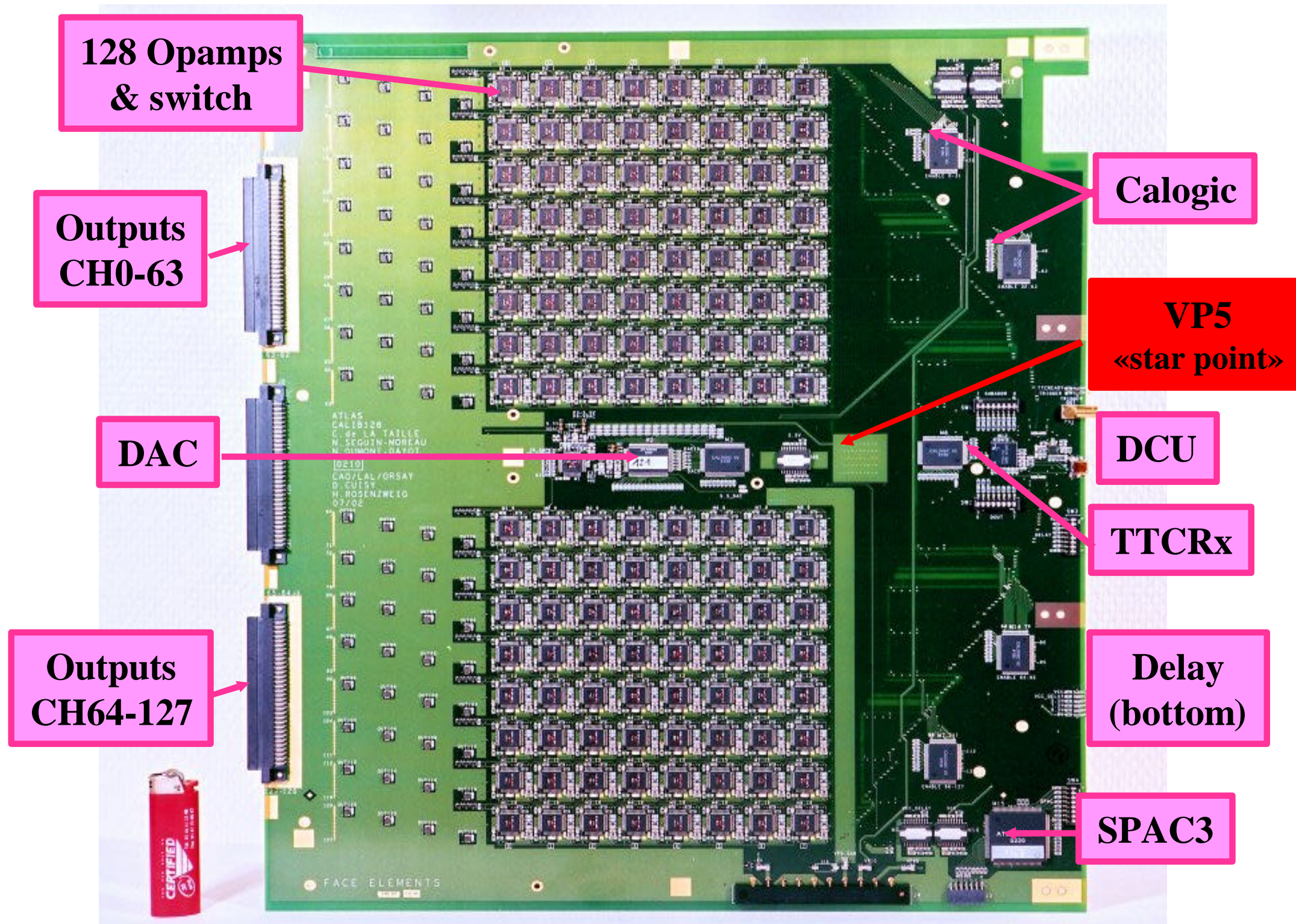


DC output current : $I_{C A L}$

Line arity on the 3 shaper ranges

- High gain $\mathcal{H G}=G 100$ :

$$
\mathcal{D A C}=0-655 \quad(0.10 \mathrm{mV})
$$

- Medium gain $M G=G 10$ :

$$
\mathcal{D A C}=0-6535 \quad(0-100 \mathrm{mV})
$$

- Lowgain: $L G=G 1$ :

$$
\mathcal{D A C}=0-65535 \quad(0-1 \mathcal{V})
$$

Line arity < $100 \mathrm{ppm}(0.01 \%)$

- $\mathcal{H G}:< \pm 1 \mu \mathcal{V}(0.07 \mathrm{LSB})$ rms $58 \mathrm{ppm}$

- $M G< \pm 10 \mu \mathcal{V}(0.7 \mathrm{LSB})$ rms $85 \mathrm{ppm}$

- $L \mathcal{G}< \pm 50 \mu \mathcal{V}(3 \mathcal{L S B})$ rms $28 \mathrm{ppm}$

- Dominated 6y $\mathcal{D A C}$ line arity
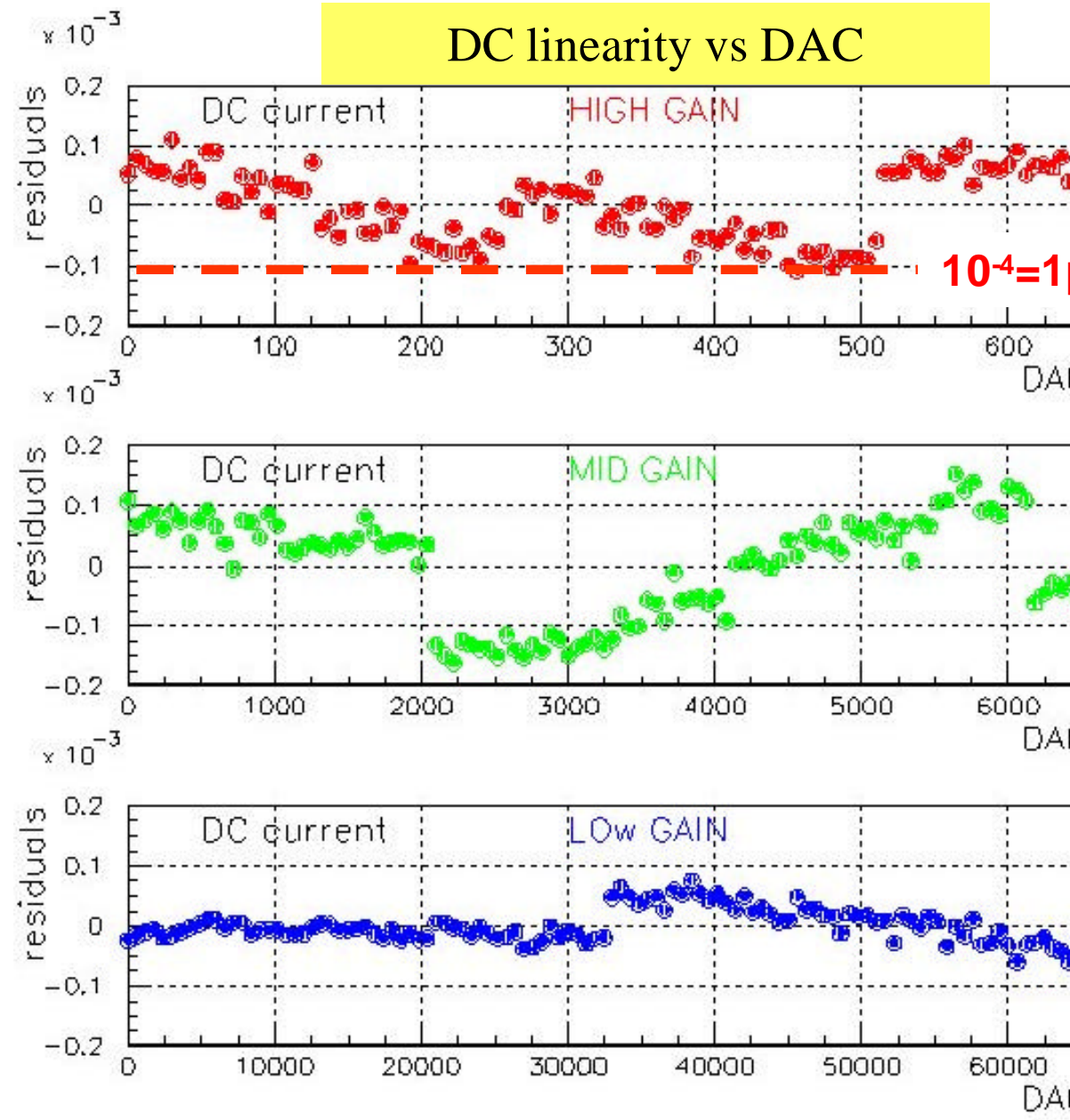

\begin{tabular}{|l|l|l|l|}
\hline IDC/DAC & P0 & P1 & RMS \\
\hline High Gain & $2.5 \mu \mathrm{A}$ & $3.0080 \mu \mathrm{A} / \mathrm{DAC}$ & $58 \mathrm{ppm}$ \\
\hline Mid Gain & $7.1 \mu \mathrm{A}$ & $3.0056 \mu \mathrm{A} / \mathrm{DAC}$ & $85 \mathrm{ppm}$ \\
\hline Low Gain & $6.7 \mu \mathrm{A}$ & $3.0056 \mu \mathrm{A} / \mathrm{DAC}$ & $28 \mathrm{ppm}$ \\
\hline
\end{tabular}


$\mathcal{D A C}=0$ : offset dominated

- $\mathcal{A} \mathcal{V G}=4.5 \mu \mathcal{A}=1.5 \mathcal{L S B}$

- $R M S=2.2 \mu \mathcal{A}=0.7 \mathcal{L S B}$

$\mathcal{D A C}=655$ (full scale $\mathcal{H} G$ )

- Without offset correction

- $\mathcal{A V G}=1975 \mu \mathcal{A}$

- $R M S S=2.7 \mu \mathcal{A}=0.9 \mathcal{L S B}$

- With offset correction

- $\mathcal{A V G}=1971 \mu \mathcal{A}$

- $R M S=1.21 \mu \mathcal{A}=0.06 \%$

$\mathcal{D A C}=6553$ (full scale $\mathcal{M G}$ )

- $\mathcal{A V G}=19.71 \mathrm{~mA}$

- $R M S=13.6 \mu \mathcal{A}=0.06 \%$

- Dominated 6y dispersion on $500.1 \%$ resistor
DC uniformity $128 \mathrm{ch}$
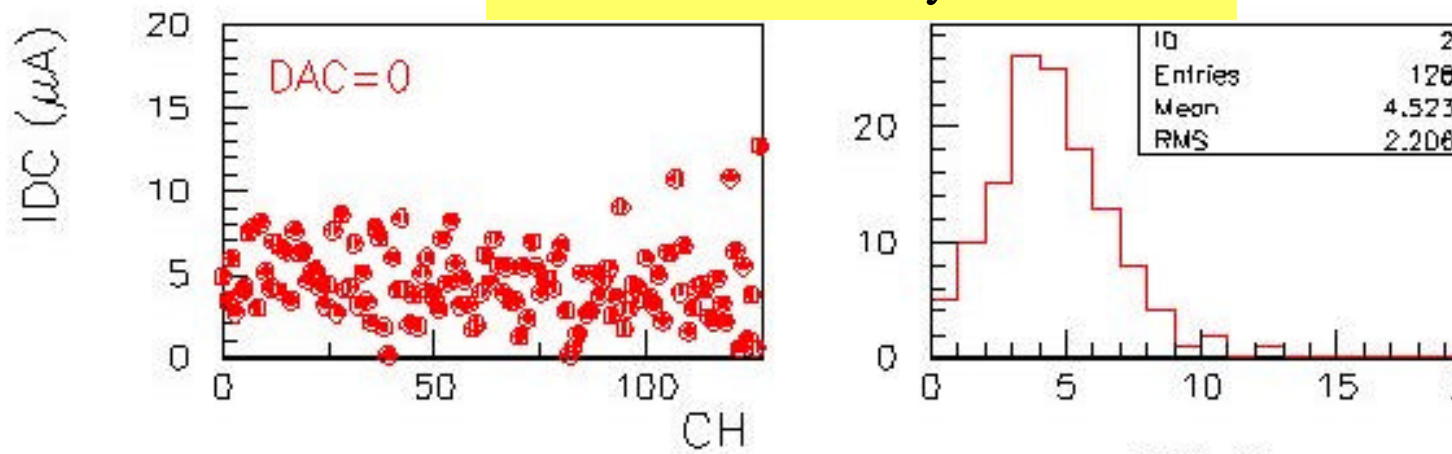

$\mathrm{DAC}=\mathrm{O}$
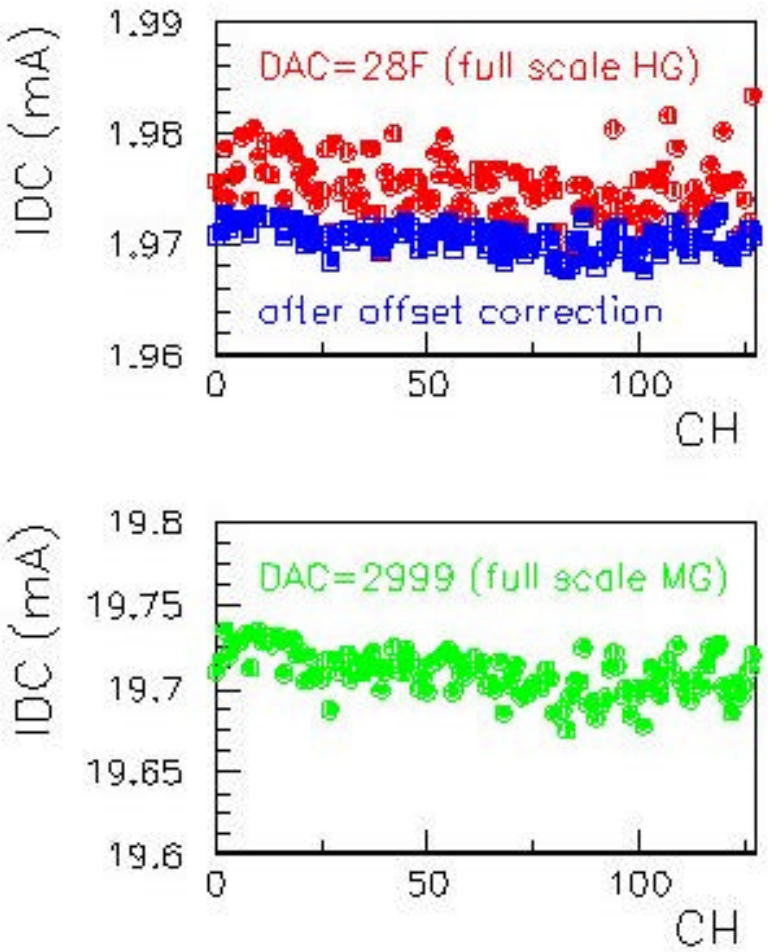

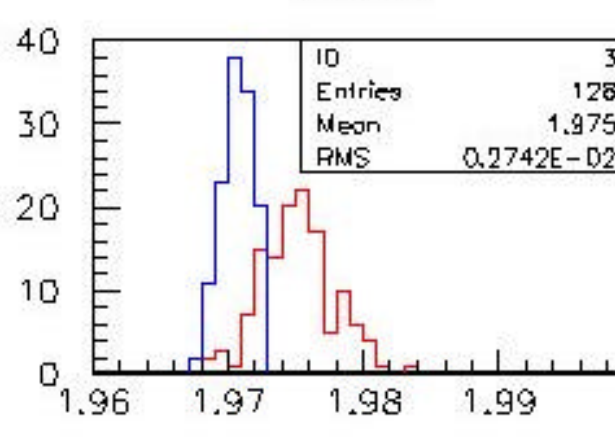

$\mathrm{DAC}=28 \mathrm{~F}$

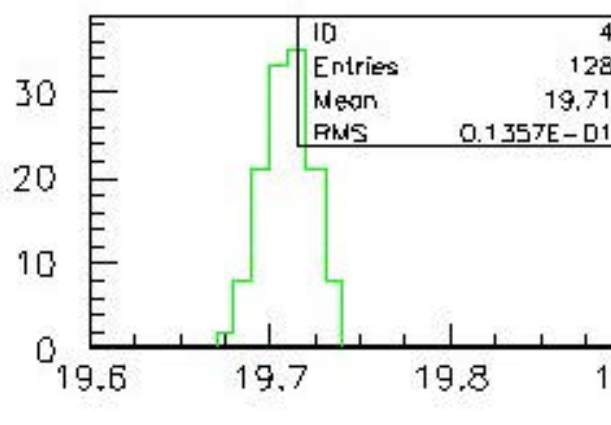

$D A C=2999$ 
Full $\mathcal{D} \mathcal{A C}$ range

- $100 \mu \mathcal{V} \rightarrow 1 \mathcal{V}$

- Ulp to $5 \mathcal{V}$ pulses in 500

Rise time $<2 n s$

- Small increase at large $\mathcal{D A C}$

Decay time 450 ns

- Matched to Argon drift time

- Accuracy: $\pm 2 \%$

- $\mathcal{H F}$ Ringings :

- At small $\mathcal{D A C}$ values, due to parasitic package inductance in $\mathcal{H F}$ switch

- "Parasitic injected charge"

- $20 \mathrm{mV} p k \cdot p \kappa$

- Very small area
Pulse output without shaping

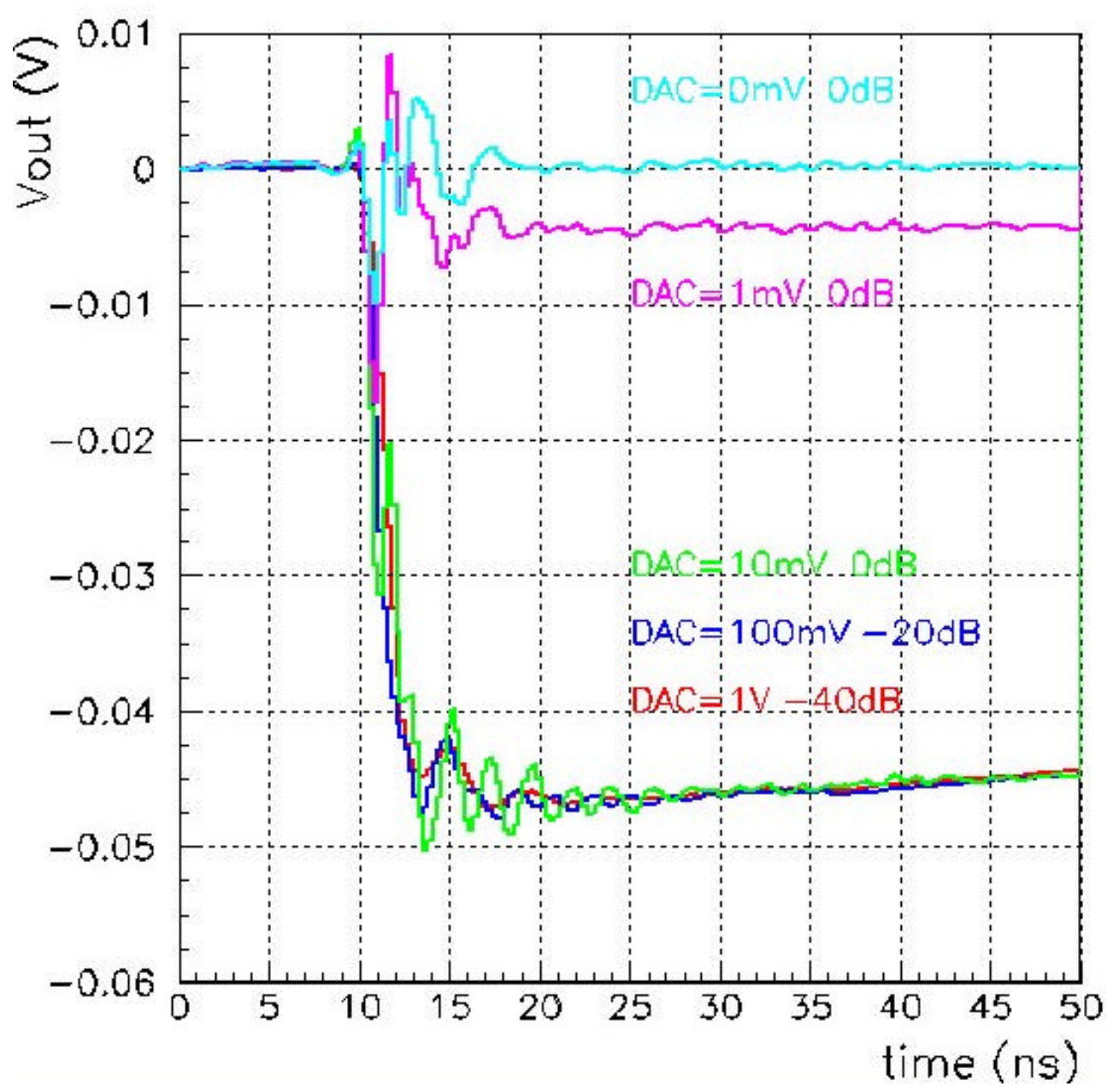


Parasitic injected charge (PIC)

- Peak of Qinj: equivalent to $\mathcal{D A C}=30 \mu \mathcal{V}(2 \mathcal{L S B})$

- At signal peak:

$\mathcal{P I C}<\mathcal{D A C}=15 \mu \mathcal{V}=1 \mathcal{L S B}$ ( 30 MeV in Barrel Middle <noise)

- Improvement $6 y>10$ compared to module 0

$C \mathcal{M D}$ feedthrough

- Parasitic pulse on disabled channels

- Equivale nt to $\mathcal{D A C}=3 \mu \mathcal{V}=0.2$ $\mathcal{L S B}$ : ne gligible

Pulse output after 50 ns shaping

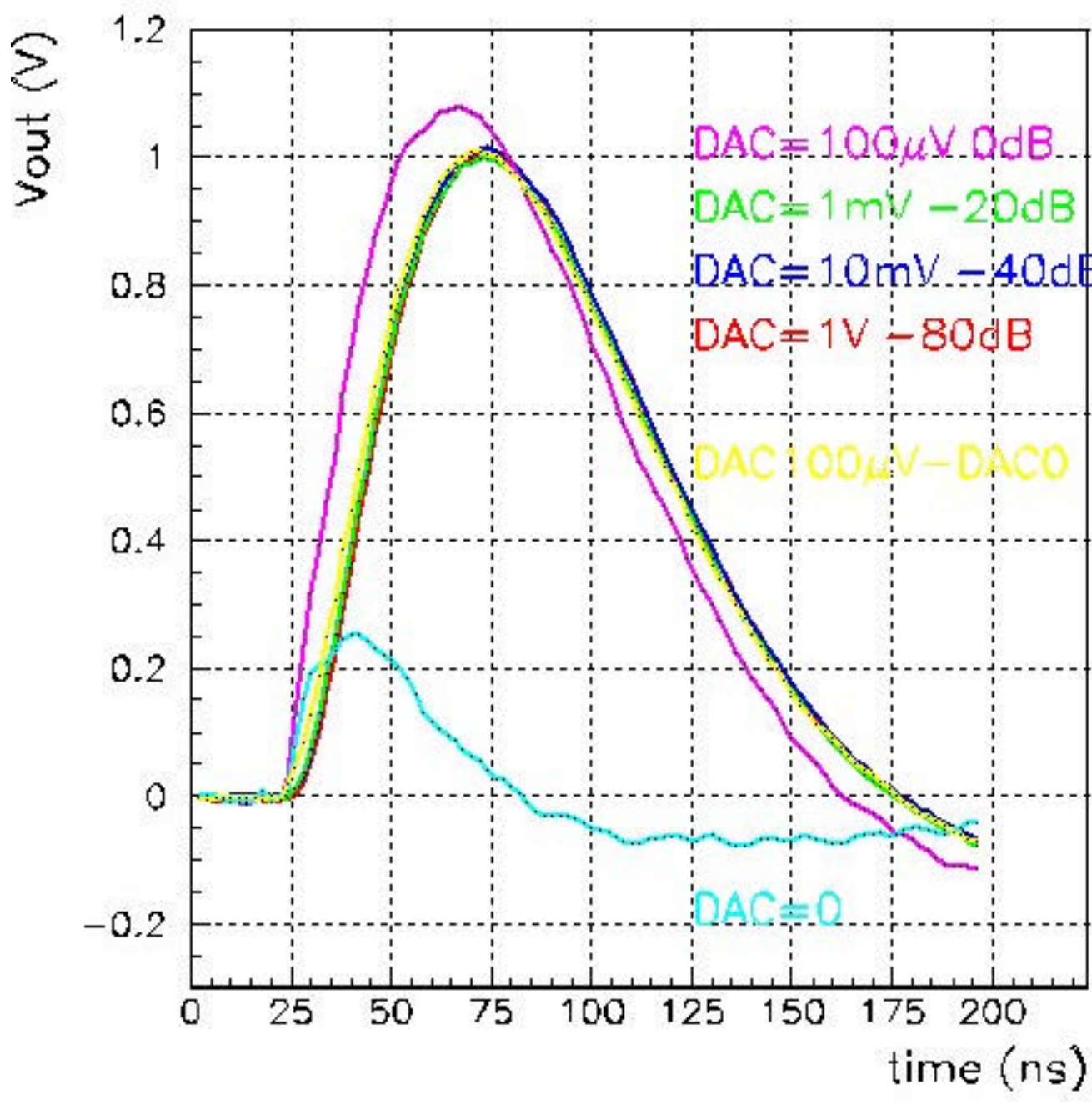


Line arity : < $0.1 \%$

- Red : at signal peak

- Black: peak of signal

- Dominated by readout non-line arity

Uniformity at $\mathcal{D A C}=5000$

- Rms : $0.13 \%$ $(\mathcal{D C}$ was $0.07 \%)$

- Additionnal contribution from output resistors, output lines, inductors and scanner board

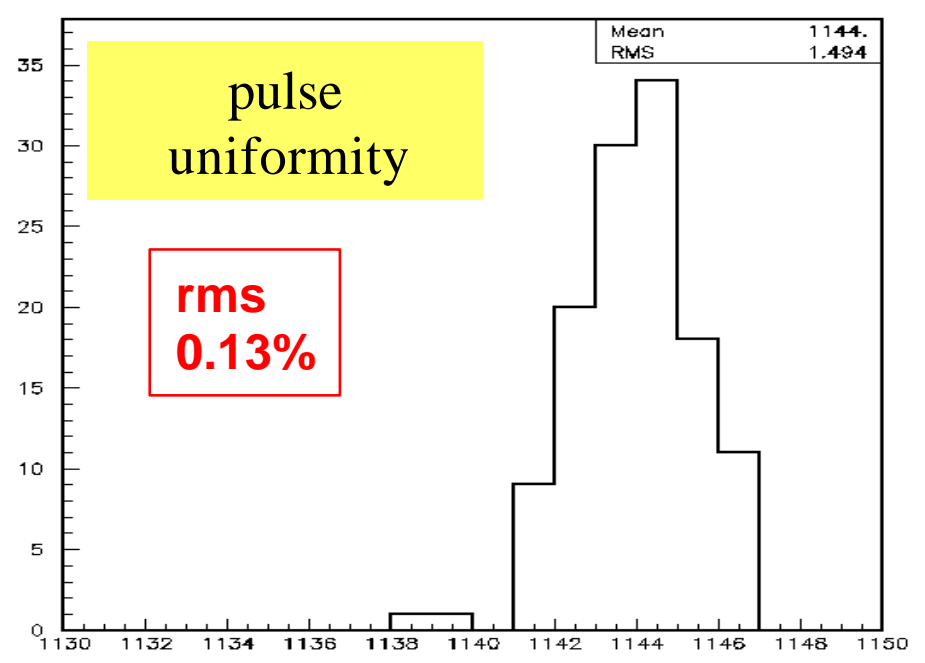

pulse linearity vs DAC
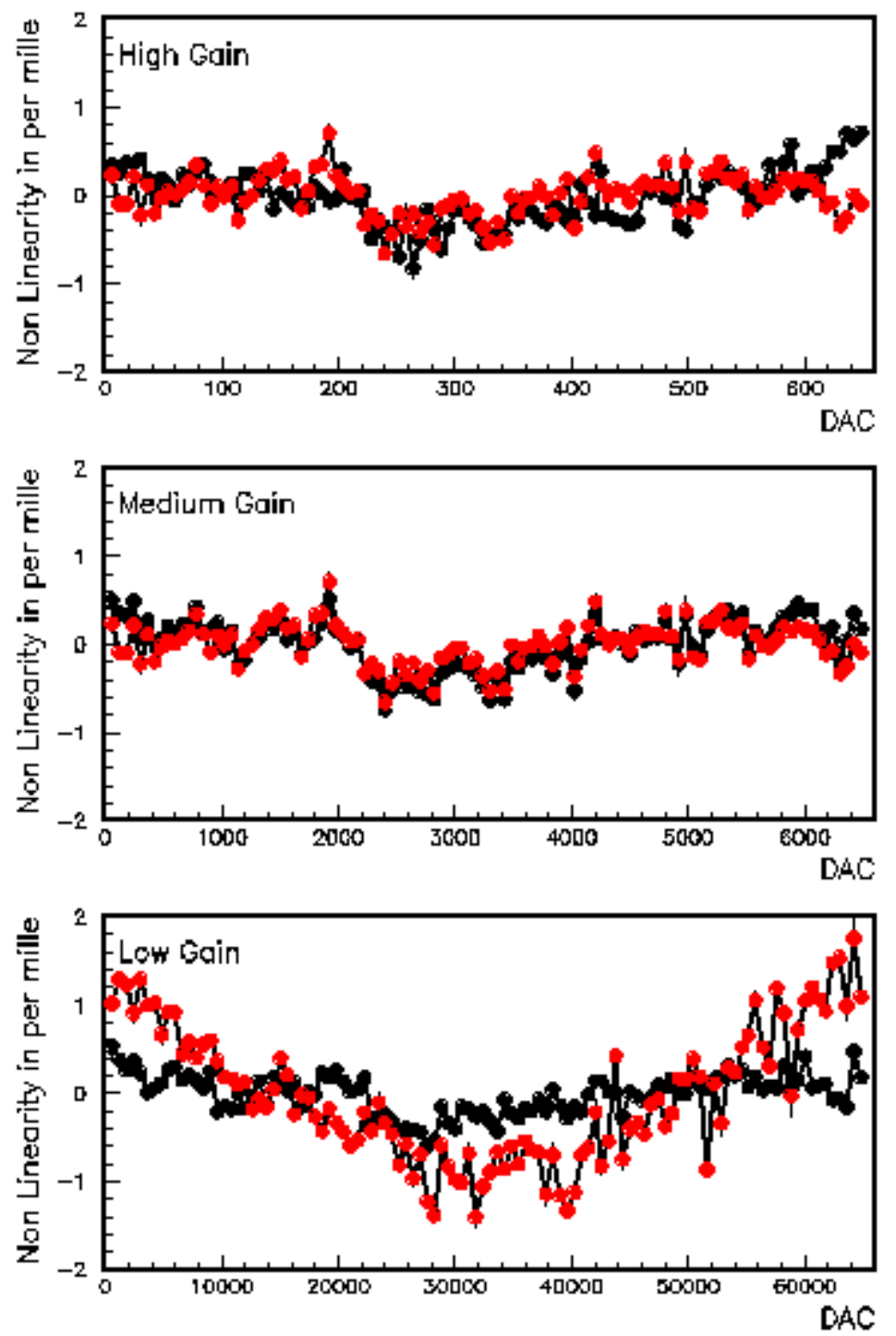
- gitter of output pulse < $75 \mathrm{ps}$

- Diminated by $\mathcal{T} \mathcal{T} C \mathcal{R}$ chip

De lay chip (PHOS 4)

- Zlsed to adjust timing be twe en calibration pulses and particles with 25 steps of $\sim 1 \mathrm{~ns}$

- Linearity : residuals within 50 ps

- Slope: varies with channel inside chip (by up to $\pm 10 \%$ )

\section{Delay linearity}

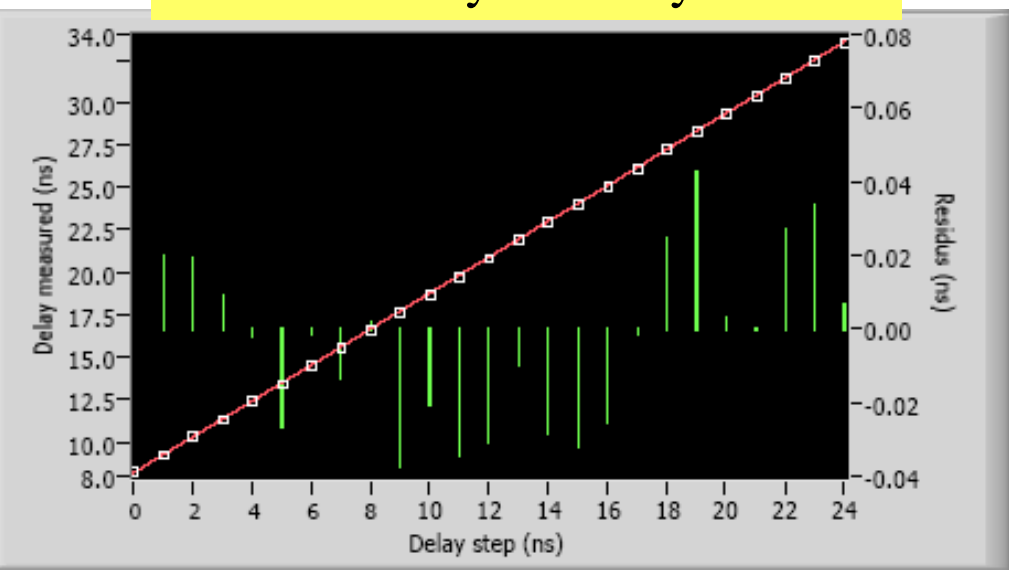

\section{Output pulse jitter vs channel\#}

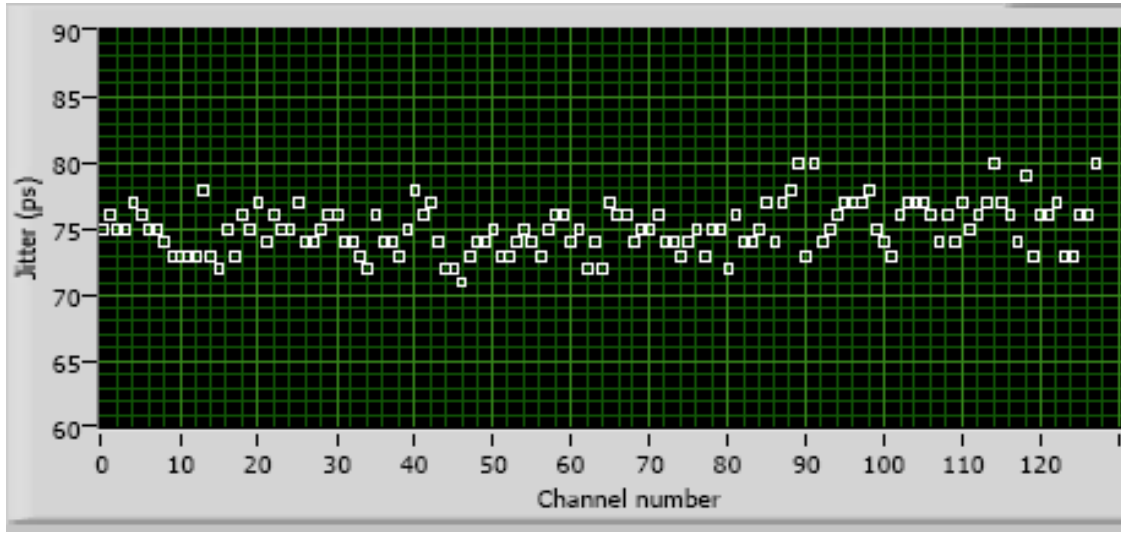

Delay step vs channel\#

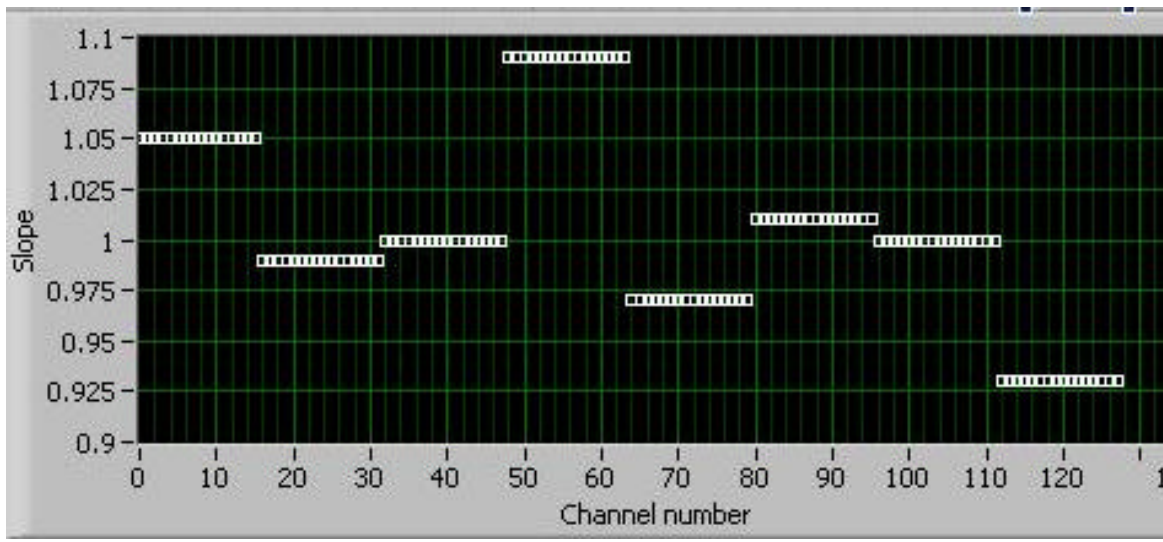


- Sensitivity to cable characteristic impedance $Z c$

- Second order effect (if terminated both ends): $d \mathcal{V} / \mathcal{V}=1-\left(d Z_{c} / 2 Z_{c}\right)^{2}$

- \pm 2.50 tole rance on cable gives $\pm 0.1 \%$

- Sensitivity to skin effect

- First order effect:

- - $1.2 \% / m @ 300 \mathcal{K}$

- $0.5 \% / m @ 77 \mathcal{K}$

- Correction necessary for cable length

- Calibration cable length:3-6 m: expect $\sim 0.2 \%$ contribution at cold ( $\sim 0.4 \%$ at warm)
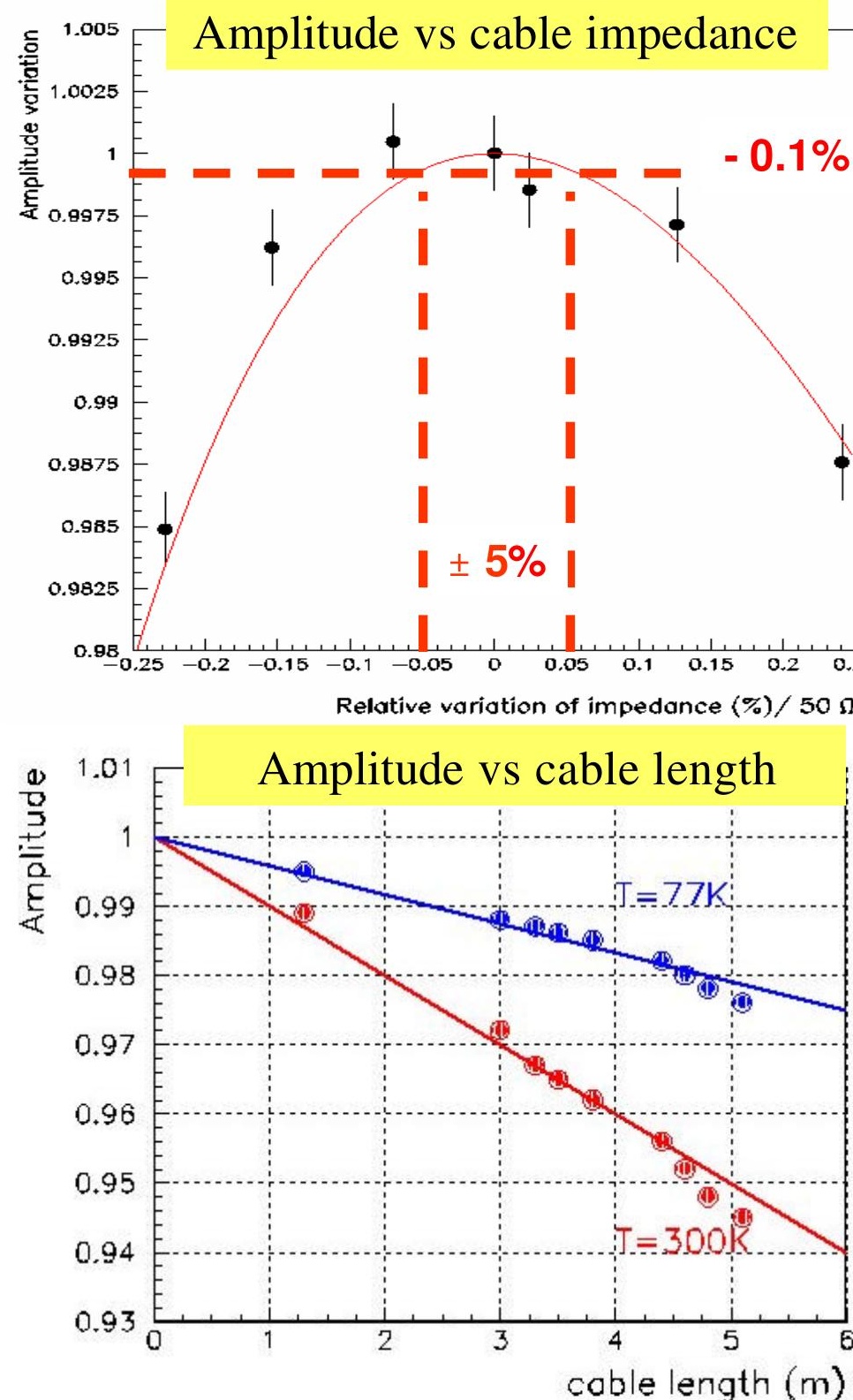


\section{Calibration at cable output}

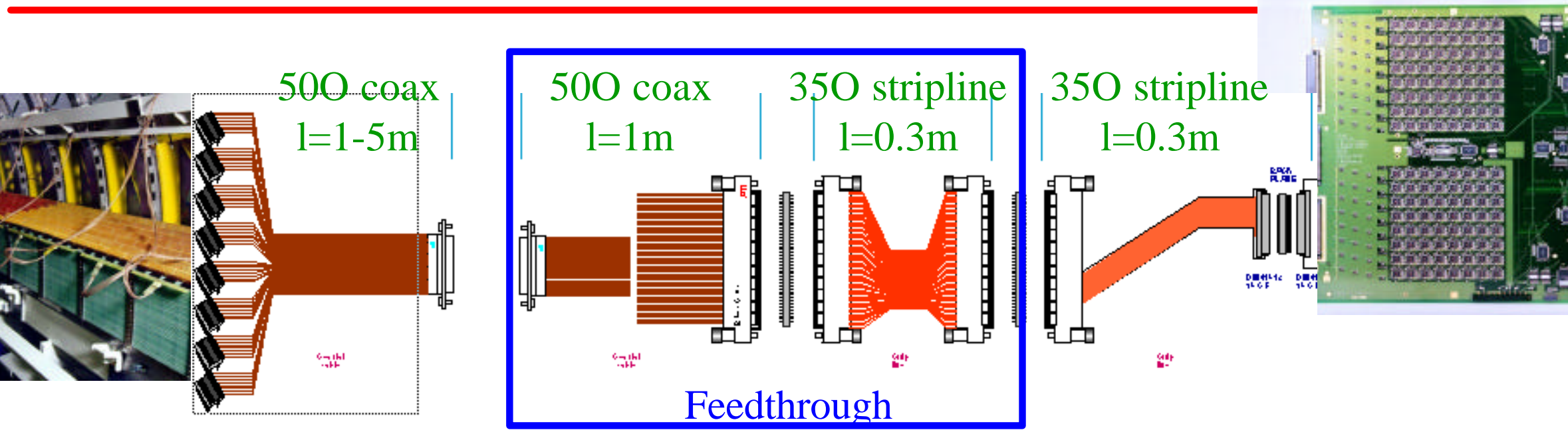

Calibration pulse at cable output

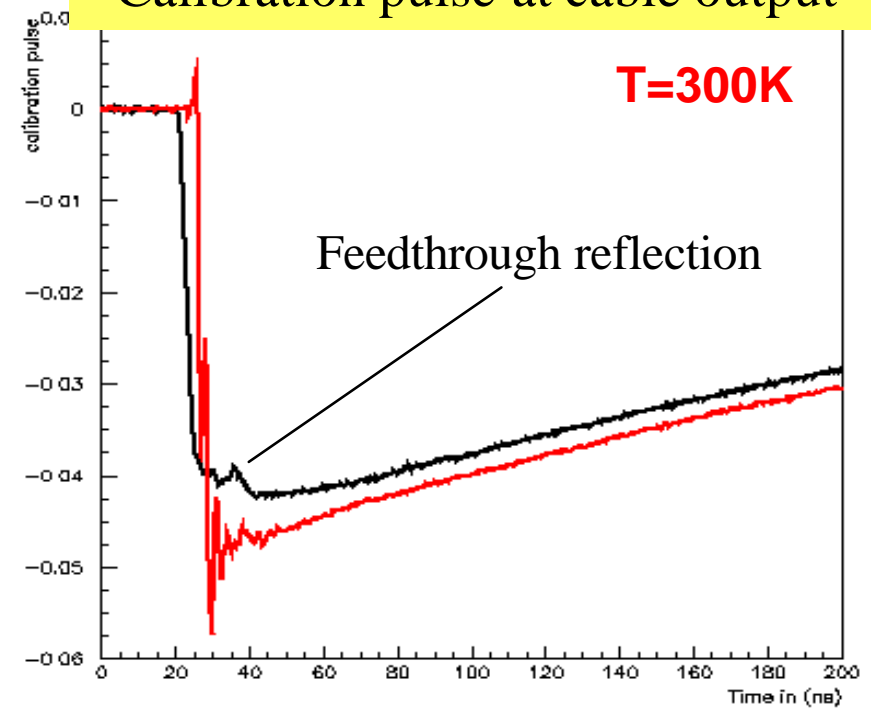

pulse uniformity at cable output

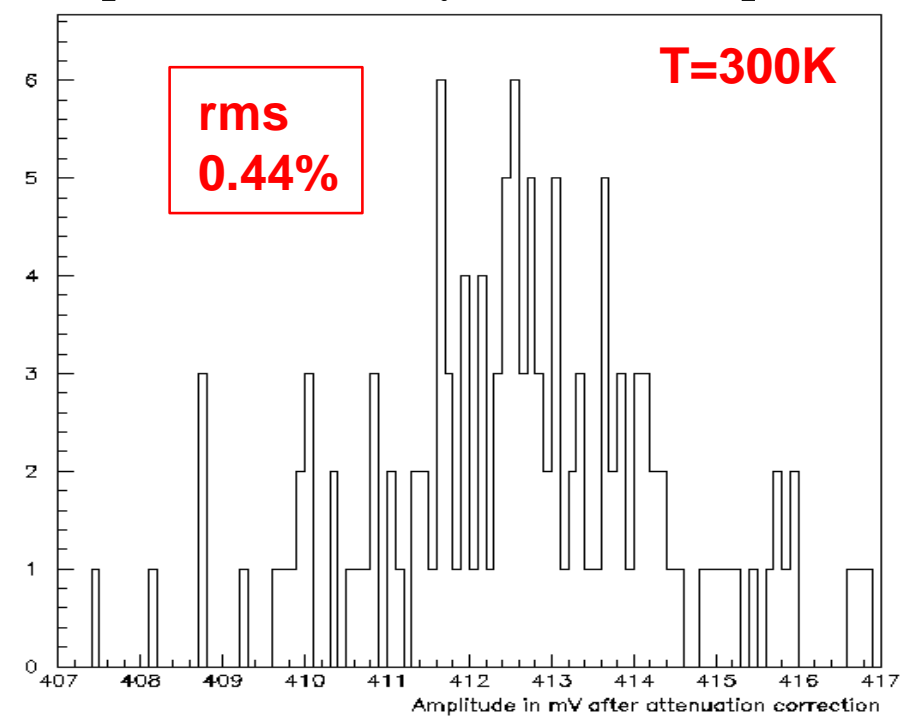


Calibration pulse shape

- Exponential shape vs triangle

- Systematic effect in $t_{S \text { HAPER }} / t_{C A L}$

- Accuracy in calib decay time $t_{C A L} \pm 2 \%$

Detector inductance

- Physics signal at shower max in the middle of the accordion: non negligible output line : inductive effect

- Sizeable effect :- $0.2 \% / n \mathcal{H}$ on

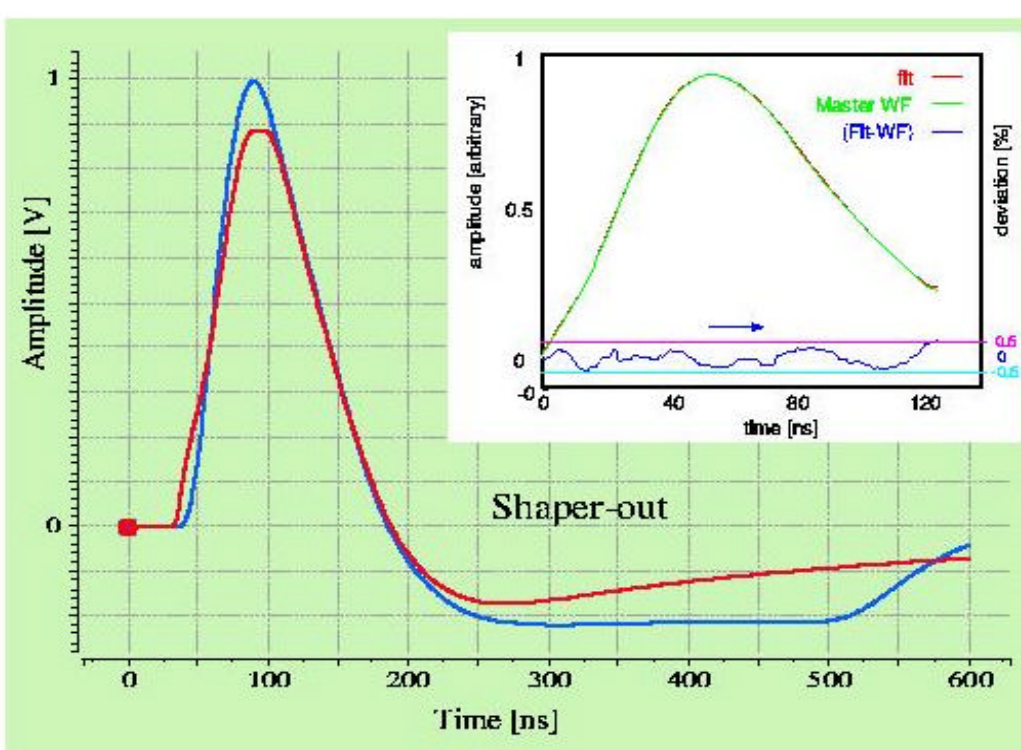
physics /calibration ratio

- Inductance measurement necessary
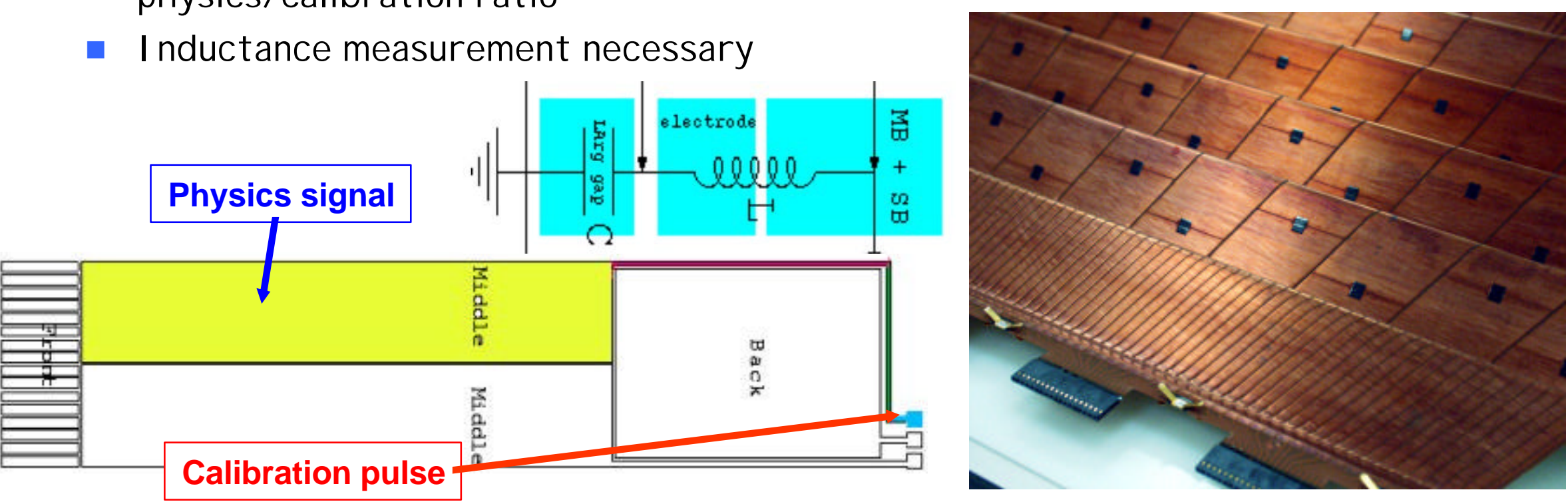


\section{Line model}

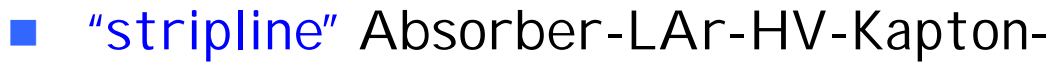
$S$ ignal. Propagation $t_{d}=4.12 \mathrm{~ns} / \mathrm{m}$

- Solving Poisson to calculate capacitances $C d, C x$ and impedance : $Z_{c}=t_{d} / C t$

Good lumped model

- Detector $\left(Z_{c}=1.5-20\right)=$ capacitance (1- $1.5 n \mathcal{F})$

- Connection $\left(Z_{c}=15-200\right)=$ inductance $(20-30 n \mathcal{H})$

- (Difficult) measurement of $f_{0}=1 / 2$ pvLC
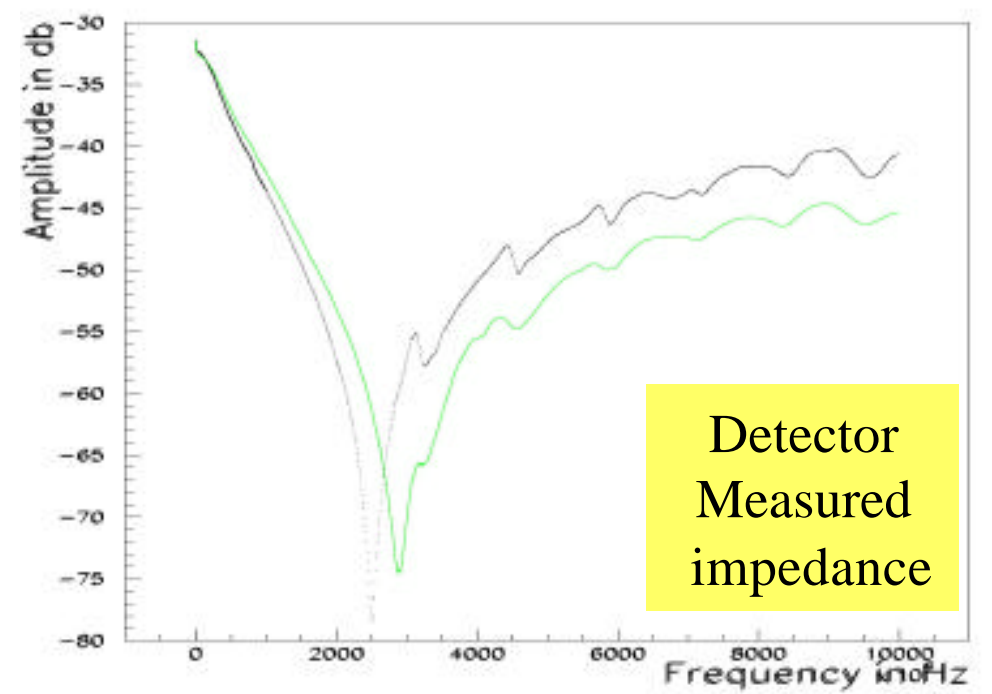

Absorber

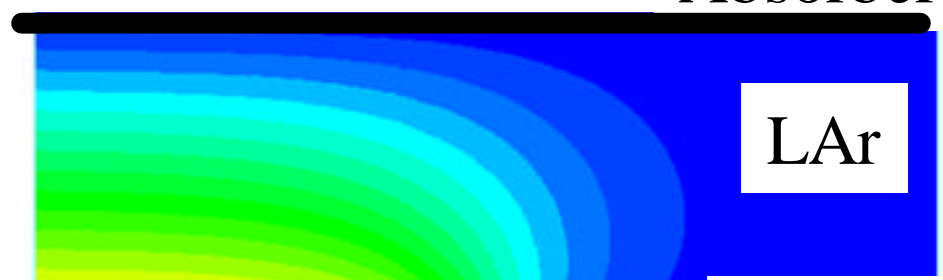

HV el.

\section{Signal electrode}

Neighbor

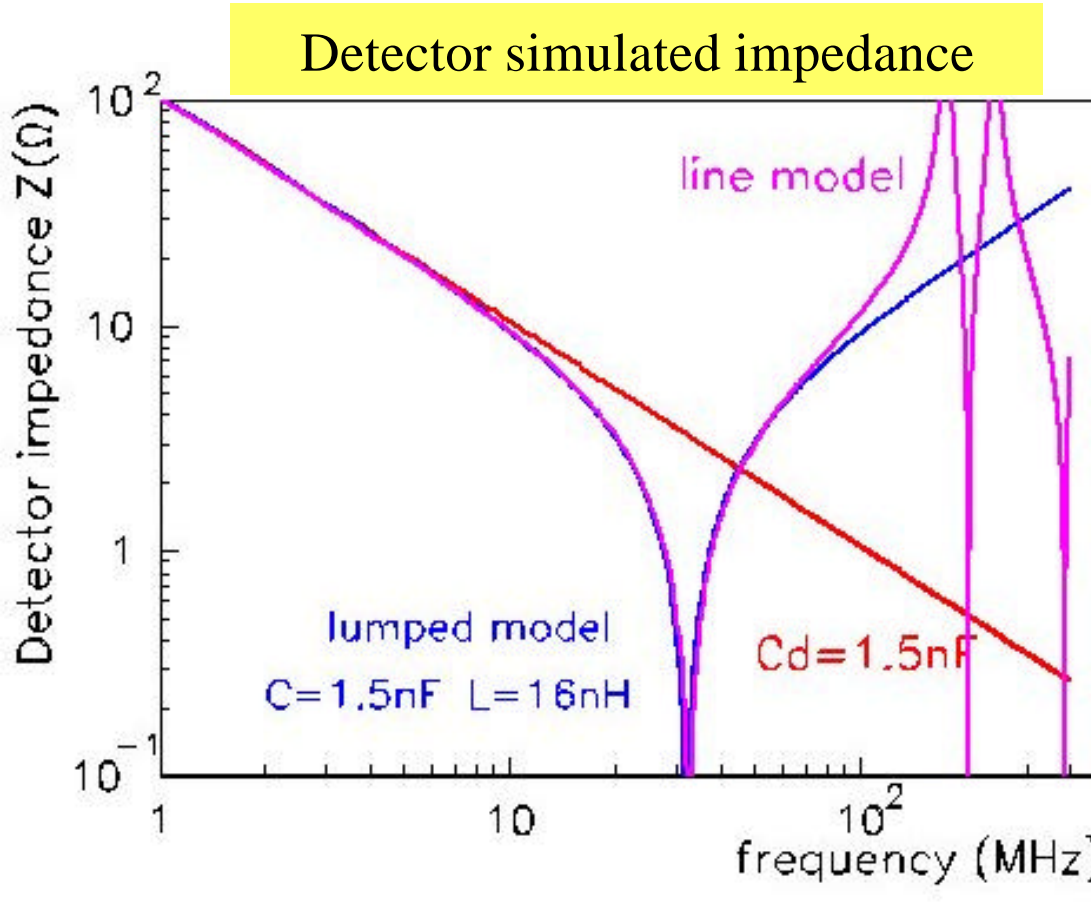


- Calibration board for $\mathcal{A} \mathcal{T} \mathcal{L} \mathcal{S}$ Lar calorime ter final

- 16 bits dynamic range : $100 \mu \mathcal{V}-5 \mathcal{V}$ pulses

- Linearity better than $100 \mathrm{ppm}$

- Board uniformity $<0.2 \%$

- Overall uniformity $<0.3 \%$

- Jitter $<100 \mathrm{ps}$

- Radiation hard

- Production of 140 boards in 2004

- DMILL AS ICS all produced

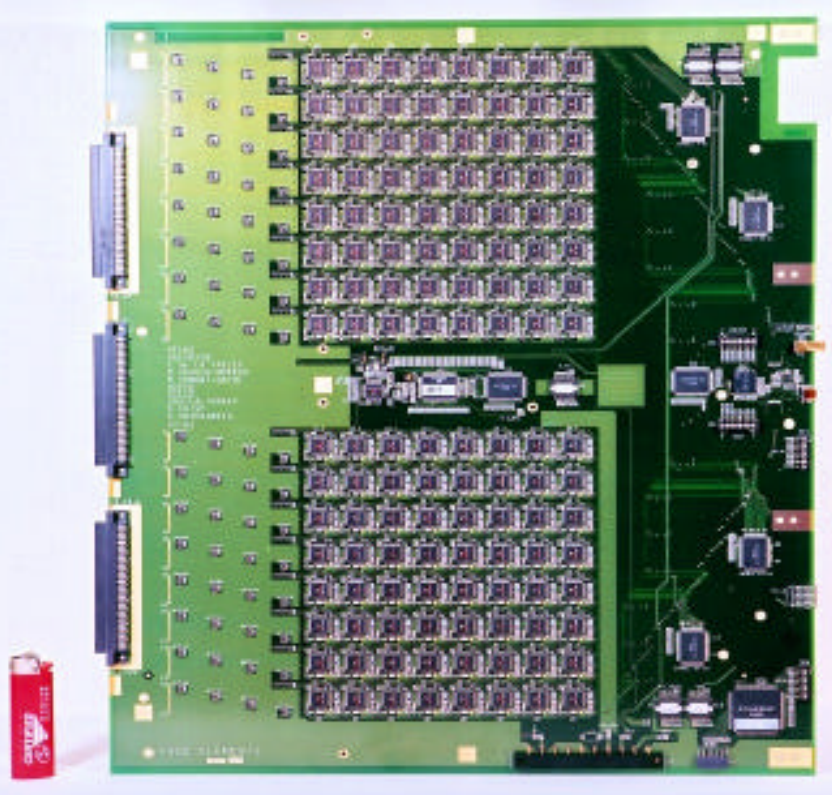

- Final prototype validated

- Installation beginning of 2005

- Calibration of calorimeter needs additionnal inputs

- Fine effects due to detector parasitic inductance need to be corrected for

- $\mathcal{A}$ major activity in 2002-2003

- See talks by L.Serin and O. Gaumer 